\title{
Simplified in vitro engineering of neuromuscular junctions between rat embryonic motoneurons and immortalized human skeletal muscle cells
}

This article was published in the following Dove Medical Press journal: Stem Cells and Cloning:Advances and Applications

\author{
Jasdeep Saini' \\ Alessandro Faroni ${ }^{2,3}$ \\ Marwah Abd Al Samid' \\ Adam J Reid ${ }^{2,3}$ \\ Adam P Lightfoot' \\ Kamel Mamchaoui ${ }^{4}$ \\ Vincent Mouly ${ }^{4}$ \\ Gillian Butler-Browne ${ }^{4}$ \\ Jamie S McPhee 5 \\ Hans Degens ${ }^{1,6,7}$ \\ Nasser Al-Shanti' \\ 'Musculoskeletal Science \& Sports \\ Medicine Research Centre, School \\ of Healthcare Science, Manchester \\ Metropolitan University, Manchester, \\ UK; ${ }^{2}$ Blond Mclndoe Laboratories, \\ Division of Cell Matrix Biology and \\ Regenerative Medicine, School of \\ Biological Sciences, Faculty of Biology \\ Medicine and Health, University of \\ Manchester, Manchester Academic \\ Health Science Centre, Manchester, \\ UK; ${ }^{3}$ Department of Plastic Surgery \\ \& Burns, University Hospitals of \\ South Manchester, Manchester \\ Academic Health Science Centre, \\ Manchester, UK; ${ }^{4}$ Center for Research \\ in Myology, Sorbonne Université- \\ INSERM, Paris, France; ${ }^{5}$ Department \\ of Sport and Exercise Science, \\ Manchester Metropolitan University, \\ Manchester, UK; ${ }^{6}$ Institute of Sport \\ Science and Innovations, Lithuanian \\ Sports University, Kaunas, Lithuania; \\ ${ }^{7}$ University of Medicine and Pharmacy \\ of Targu Mures, Targu Mures, Romania
}

Correspondence: Jasdeep Saini School of Healthcare Science, Manchester Metropolitan University, John Dalton Building, E247, Oxford Road, Manchester, MI 5GD, UK

Tel +44016I 2475712

Email Jas.Saini2015@gmail.com
Background: Neuromuscular junctions (NMJs) consist of the presynaptic cholinergic motoneuron terminals and the corresponding postsynaptic motor endplates on skeletal muscle fibers. At the NMJ the action potential of the neuron leads, via release of acetylcholine, to muscle membrane depolarization that in turn is translated into muscle contraction and physical movement. Despite the fact that substantial NMJ research has been performed, the potential of in vivo NMJ investigations is inadequate and difficult to employ. A simple and reproducible in vitro NMJ model may provide a robust means to study the impact of neurotrophic factors, growth factors, and hormones on NMJ formation, structure, and function.

Methods: This report characterizes a novel in vitro NMJ model utilizing immortalized human skeletal muscle stem cells seeded on $35 \mathrm{~mm}$ glass-bottom dishes, cocultured and innervated with spinal cord explants from rat embryos at ED 13.5. The cocultures were fixed and stained on day 14 for analysis and assessment of NMJ formation and development.

Results: This unique serum- and trophic factor-free system permits the growth of cholinergic motoneurons, the formation of mature NMJs, and the development of highly differentiated contractile myotubes, which exhibit appropriate configuration of transversal triads, representative of in vivo conditions.

Conclusion: This coculture system provides a tool to study vital features of NMJ formation, regulation, maintenance, and repair, as well as a model platform to explore neuromuscular diseases and disorders affecting NMJs.

Keywords: neuromuscular junction, NMJ, coculture, myoblast, myotube, motor neuron, motoneuron

\section{Introduction}

Although the neuromuscular junction (NMJ) plays a central role in the pathology of neuromuscular (NM) disorders, current methods to study its role in NM pathology have severe limitations. For instance, most studies on NM diseases rely on in vivo animal models that do not entirely reflect disease in humans. ${ }^{1}$ Shortcomings of in vitro models of NM disorders are that they are largely based on cells derived from animals, 2,3 or skeletal muscle cell (SkMC) culture systems that fail to mimic in vivo conditions, particularly due to the lack of functional innervation. ${ }^{4}$ Thus, the development of new models to study and manipulate NMJs has the potential to provide significant insight into NM disease pathogenesis and detection, and to test the efficacy of innovative therapies.

To fill this gap, a small number of promising nerve-muscle coculture models have been developed using mouse, rat, primary human myoblasts, human embryonic

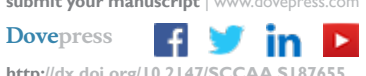


stem cell (hESC) and human induced pluripotent stem cell (hiPSC)-derived cells, and cross-species systems. ${ }^{5-9}$ However, some previously established coculture systems suffer from poor experimental reproducibility due to the complexity of the culture system. For example, the use of serum in nervemuscle coculture systems introduces unspecified variables that can distort the effects of experimental treatments on the system. Further research demonstrates diminished motoneuron myelination in vitro that can be attributed to the use of serum within the culture system. ${ }^{10}$

Coculture systems utilizing primary human skeletal muscle stem cells obtained from muscle biopsies present their own distinct complications as they have limited proliferative capacity and varied cell purity, and experience phenotypic changes when expanded. The phenotypic change is further compounded by progressive cellular senescence during primary cell expansion, ${ }^{11,12}$ rendering primary myoblasts a problematic choice for a consistently reproducible coculture system. The use of hESC/hiPSC-derived cells to generate myoblasts ${ }^{13}$ and motoneurons ${ }^{14}$ may allow for the development of novel coculture systems. However, neuronal cells derived from stem cells are particularly challenging to culture, involving complex media with numerous neurotrophic factors that can have detrimental effects on SkMCs. In addition, cocultures of stem-cell-derived motoneurons and myoblasts result in poorly developed NMJs and have not been maintained for longer than 21 days, ${ }^{15}$ which is inadequate for long-duration studies.

This work set out to establish a simplified and easily reproducible nerve-muscle coculture system generating the formation of NMJs. Thus, we developed a novel coculture model devoid of serum and growth/neurotrophic factors. Immortalized human myoblasts were differentiated to mature myotubes while simultaneously being innervated with motoneurons, emanating from neonatal rat spinal cord explants. We observed that this system resulted in the development of highly contractile myotubes that exhibited acetylcholine receptor (AChR) aggregation in the typical twisting knotted structure of mature NMJs that co-localized with motoneuron axon terminals. The success of this system thus offers an easy and reproducible bridge between animal and clinical studies of NM disease and may well serve as a platform to screen the efficacy of novel therapeutic agents.

\section{Experimental procedures Immortalized human SkMC culture}

A non-commercial immortalized human skeletal muscle cell (SkMC) line was generated at the Institute of Myology
(Paris, France). The cell line was established using primary human myoblasts obtained anonymously from Myobank, a tissue bank affiliated to Eurobiobank, which has an agreement from the French Ministry of Research (authorization number AC-2013-1868). The primary myoblasts originated from biopsies of the semitendinosus muscle of a 25 -year-old man, free of genetic defects and disease. Myoblast immortalization was achieved using transduction with both telomerase-expressing and cyclin-dependent kinase 4-expressing vectors. ${ }^{37}$ A 1-mL frozen vial containing a suspension of $1 \times 10^{6} \mathrm{SkMCs}$ in $90 \% \mathrm{FBS}$ and $10 \%$ dimethyl sulfoxide (DMSO) was thawed and resuspended in $10 \mathrm{~mL}$ of complete growth media $(\mathrm{GM})^{37}$ to induce SkMC proliferation.

Cells were incubated at $37^{\circ} \mathrm{C}$ with a $5 \% \mathrm{CO}_{2}$ atmosphere until $80 \%$ confluent. Subsequently, cells were washed twice with Dulbecco's phosphate-buffered saline (DPBS) (Lonza, Basel, Switzerland). Cells were disassociated using $2 \mathrm{~mL}$ of TrypLE ${ }^{\mathrm{TM}}$ Express Enzyme (Thermo Fisher Scientific, Waltham, MA, USA) and incubated at $37^{\circ} \mathrm{C}$ in $5 \% \mathrm{CO}_{2}$ for 5 minutes. The cells were counted and then seeded on a 35-mm glass-bottom $\mu$-Dish (ibidi ${ }^{\circledR}$, Martinsried, Germany) at a density of 350 cells $/ \mathrm{mm}^{2}$, and incubated at $37^{\circ} \mathrm{C}$ with a $5 \% \mathrm{CO}_{2}$ atmosphere for 24 hours.

Following incubation in GM, cells were washed twice with DPBS and switched to a simplified differentiation medium (DM) consisting of 99\% (v/v) DMEM, 1\% (v/v) L-glutamine, $10 \mu \mathrm{g} / \mathrm{mL}$ recombinant human insulin, and 10 $\mu \mathrm{g} / \mathrm{mL}$ gentamicin. The cells were incubated for 24 hours in $\mathrm{DM}$ at $37^{\circ} \mathrm{C}$ with a $5 \% \mathrm{CO}_{2}$ atmosphere before plating the rat embryo spinal cord explants.

\section{Isolation of rat embryonic spinal cord explants}

Ethical approval for the animal work was obtained from the animal facility under a general S1 Home Office license at the University of Manchester. Animal welfare was in accordance with the guidelines detailed in the Animals Scientific Procedures Act 1986, which regulates the use of living vertebrates and cephalopods in scientific procedures within the UK. Time-mated Sprague-Dawley rats from Charles River (Oxford, UK) were killed with $\mathrm{CO}_{2}$ when embryos were between embryonic development day 13 and 14. Embryo dissection was performed in a 100-mm dish under a binocular microscope using 21-gage needles. The spinal cord was dissected in one piece from the embryo and surrounding connective tissue removed, ensuring that the dorsal root ganglia (DRGs) remained attached to the spinal cord. The spinal cord was sliced transversally into $\sim 1-2 \mathrm{~mm}^{3}$ explants. 


\section{Coculture}

DM was removed from the dishes and the cells were washed twice with DPBS; $500 \mu \mathrm{L}$ DM was added to each dish. Five evenly spaced explants were placed in each dish and incubated for 3 hours at $37^{\circ} \mathrm{C}$ with a $5 \% \mathrm{CO}_{2}$ atmosphere. Once explants were slightly attached to the SkMCs, an additional $500 \mu \mathrm{L}$ DM was added to each dish and then incubated for 24 hours. After 24 hours of incubation, a further $1 \mathrm{~mL}$ of DM was added to each dish. As the myocytes fuse into immature myotube between 24 and 48 hours, sprouting neurites from the explants innervate the cells at this stage of development. Cocultures were maintained by changing half the DM every 48 hours. Live cells were visualized using a Leica DMI6000 B inverted microscope (Leica Microsystems, Milton Keynes, UK). In addition, real-time myotube contractions were video-captured with phase-contrast microscopy on day 7 at 24 frames per second. Cell fixation for immunocytochemistry was performed on day 14 .

\section{Immunocytochemistry}

Cells were washed twice with DPBS and fixed in 4\% paraformaldehyde for 10 minutes at $21^{\circ} \mathrm{C}$. Fixed cells were washed thrice with DPBS and permeabilized with $1 \times$ perm/ wash buffer (BD Biosciences, Franklin Lakes, NJ, USA), and incubated for 30 minutes at $21^{\circ} \mathrm{C}$, followed by a final wash with DPBS. Cells were then incubated for 1 hour in a blocking solution of $0.2 \%$ Triton X-100 with $10 \%$ normal goat serum (GS) or 10\% normal donkey serum (DS) (all from Sigma-Aldrich, St Louis, MO, USA). Blocking solution was removed and cells were washed once with DPBS. The primary antibody in a diluent consisting of $3 \%$ GS or DS with 0.05\% Tween-20 (Sigma-Aldrich, St Louis, MO, USA) was added to the cells and incubated for $18-24$ hours at $4^{\circ} \mathrm{C}$. Following primary antibody incubation, the cells were washed thrice with DPBS before incubation with the corresponding secondary antibodies for 1 hour at $21^{\circ} \mathrm{C}$. Confirmation of myotube innervation and NMJ formation was assessed via confocal microscopy using the Leica TCS SP5 confocal microscope (Leica Microsystems, Wetzlar, Germany).

\section{Quantification of NMJ morphologies}

Innervated and aneural cultures were fixed and stained as described above. Various NMJ morphologies were quantified using previously established NMJ morphology development classifications (ie, mature, fragmented, faint, premature, denervated). ${ }^{38-41}$ Twenty random fields of view were assessed at $20 \times$ magnification using the Leica TCS SP5 confocal microscope for each data set.

\section{Triad quantification}

Innervated and aneural cultures were fixed and stained as described above. Verification of triad formation was assessed using the Leica TCS SP5 confocal microscope. Thirty random fields of view were assessed at $40 \times$ magnification.

\section{Results \\ Characterization of cholinergic motoneurons}

An appropriate NMJ is characterized by the connection of cholinergic motoneurons via endplates with SkMCs. ${ }^{16}$ In our coculture, motoneurons and myotubes were identified by their staining for choline acetyltransferase (ChAT) and myosin heavy chain (MHC), respectively. Indeed, they were often co-localized (Figure 1) and, together with the numerous motoneuron axon terminals in contact with differentiated myotubes, suggest innervation of the SkMCs. There were also some myotubes with multiple points of contact with
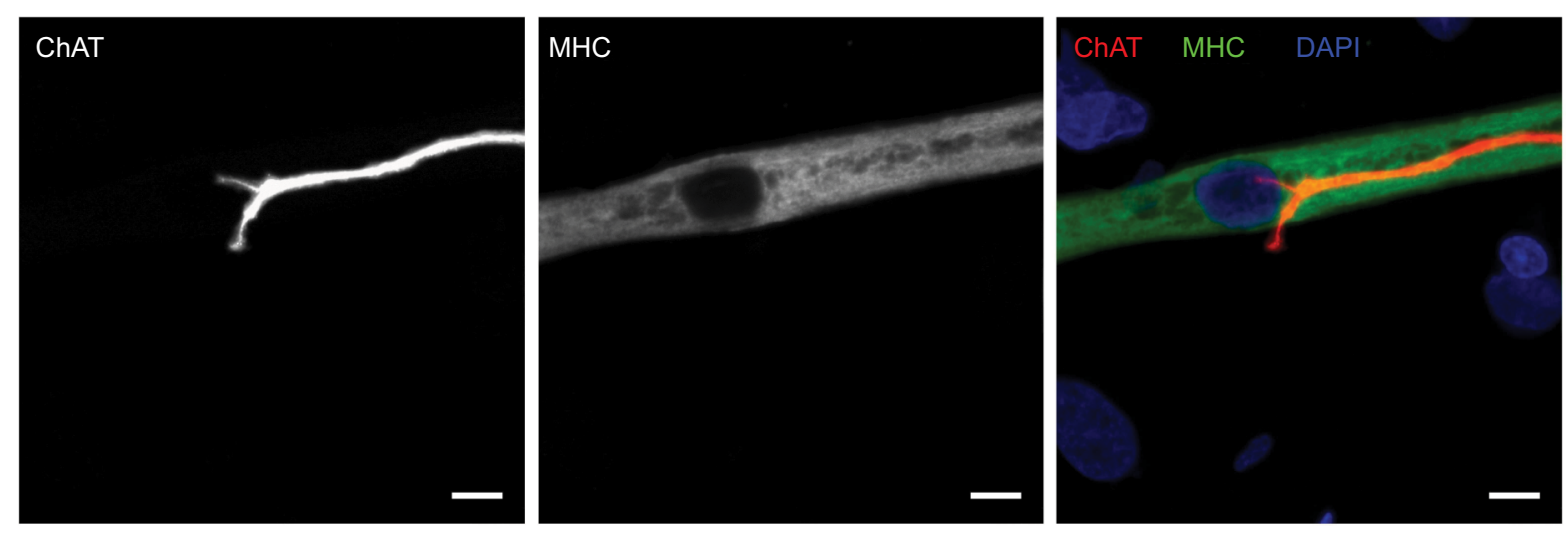

Figure I Cholinergic motoneurons co-localize with myotubes at day 14.

Notes: Representative images of human myotube cocultured with embryonic rat motoneuron stained for choline acetyltransferase (ChAT) (red), myosin heavy chain (MHC) (green), and DAPI (blue). Scale bars $=7.5 \mu \mathrm{m}$. 
axon terminals, similar to what is seen in early postnatal muscle development. During in vivo development, myotubes are innervated by multiple branching axons, which extend from many motoneurons. As development progresses, axon pruning occurs and hundreds of mature muscle fibers are innervated by single motoneurons, creating a functional motor unit. ${ }^{17}$ Importantly, the development of the cocultures appeared similar to what is observed in vivo before axon pruning occurs.

\section{NMJ formation}

Having confirmed the co-localization of cholinergic motoneurons with differentiated myotubes, subsequent experiments were performed to verify that synaptic connections were indeed formed between the motoneurons and myotubes. NMJs are distinguishable by highly clustered AChRs on the myotube membrane in close proximity of motoneuron axons. Motoneurons were identified by staining for $\beta$-III-tubulin and AChR clusters were identified with fluorescently labeled $\alpha$-bungarotoxin ( $\alpha$-BTX) (Figure 2 ). In the cocultures, numerous axon terminals co-localized with AChR aggregations on the myotube membrane. These clusters of AChRs had the typical twisting-knotted configuration, as seen in vivo, while sparse, unstructured and disjointed AChRs were observed on the membranes of myotubes that were aneurally cultured.

The efficacy of NMJ formation in the coculture system was much better than that in aneurally cultured myotubes, as reflected by a larger proportion of mature NMJs (Figure 3 ).

\section{Spontaneous myotube contractions}

The first spontaneous muscle contractions were detected in the myotubes $\sim 3$ days after co-culturing the SkMCs with rat embryo spinal cord explants. We believe this is the first time spontaneous contractions have been observed at this early stage of development in a nerve-muscle coculture system.
Despite the fact that myotube contractions were observed as early as 72 hours, only individual arrhythmically contracting myotubes were observed at this time point, with the most vigorously contracting myotubes closest to the explant. The cocultures progressively displayed an increase in both the number of contracting myotubes and the frequency of contractions. By day 7 post coculture, networks of myotubes were contracting simultaneously in a rhythmic manner (Video S1), suggesting that the myotube network functioned as a single motor unit, receiving bursts of stimulation from the motoneurons. Contracting myotubes separated from the culture plate adopted the morphological characteristics of three-dimensional tubes. Conversely, aneurally cultured myotubes did not contract and maintained a flat two-dimensional morphology, firmly fixed to the culture plate surface. These observations indicate that the observed NMJs are in fact functional. However, even at this time point, multiple innervations were still visible on a large proportion $(83.4 \pm 12.6 \%$, $\mathrm{n}=20$ ) of the innervated myotubes.

\section{Characterization of innervated myotubes}

Mature myotubes in vivo display triads that are arranged in a repetitive transversal manner. Triads consist of a T-tubule with terminal cisternae on each side. ${ }^{18} \mathrm{~T}$-tubules were identified by staining for the dihydropyridine receptor (DHPR), a voltage-dependent calcium channel located in the T-tubule membrane, ${ }^{19}$ and the terminal cisternae were identified by staining for the ryanodine receptor (RyR), which is located on the membrane of the sarcoplasmic reticulum. ${ }^{20}$ By day 14 , about $32 \%$ of the cocultured myotubes had well-developed triads (Figure 4A), whereas none of the aneurally cultured SkMCs exhibited the triad structure (Figure 4B, C).

\section{Discussion}

We developed a serum-free coculture of immortalized human myoblasts and embryonic rat spinal cord explants
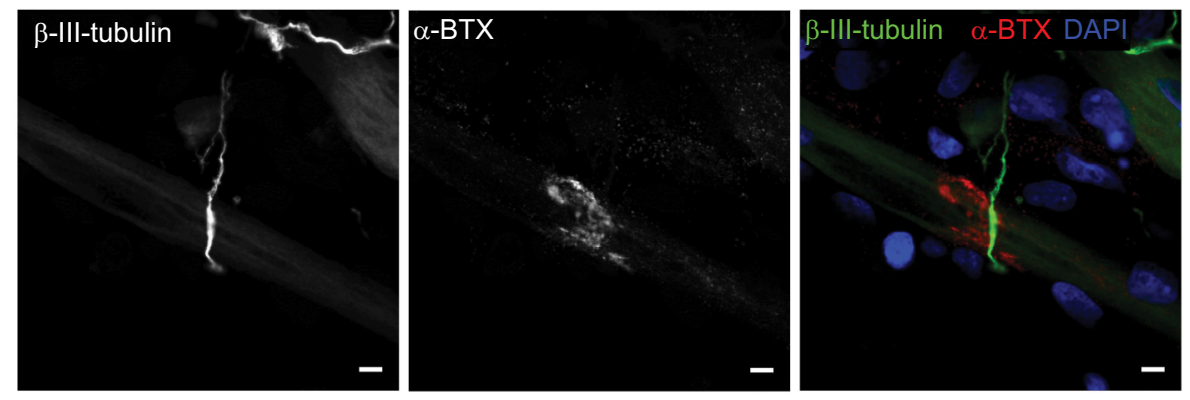

Figure 2 Characterization of neuromuscular junction formation at day 14 .

Notes: Representative images of coculture stained for $\beta$-III-tubulin (green), $\alpha$-bungarotoxin $(\alpha-B T X)$ (red), and DAPI (blue). Scale bars $=5 \mu$ m. 


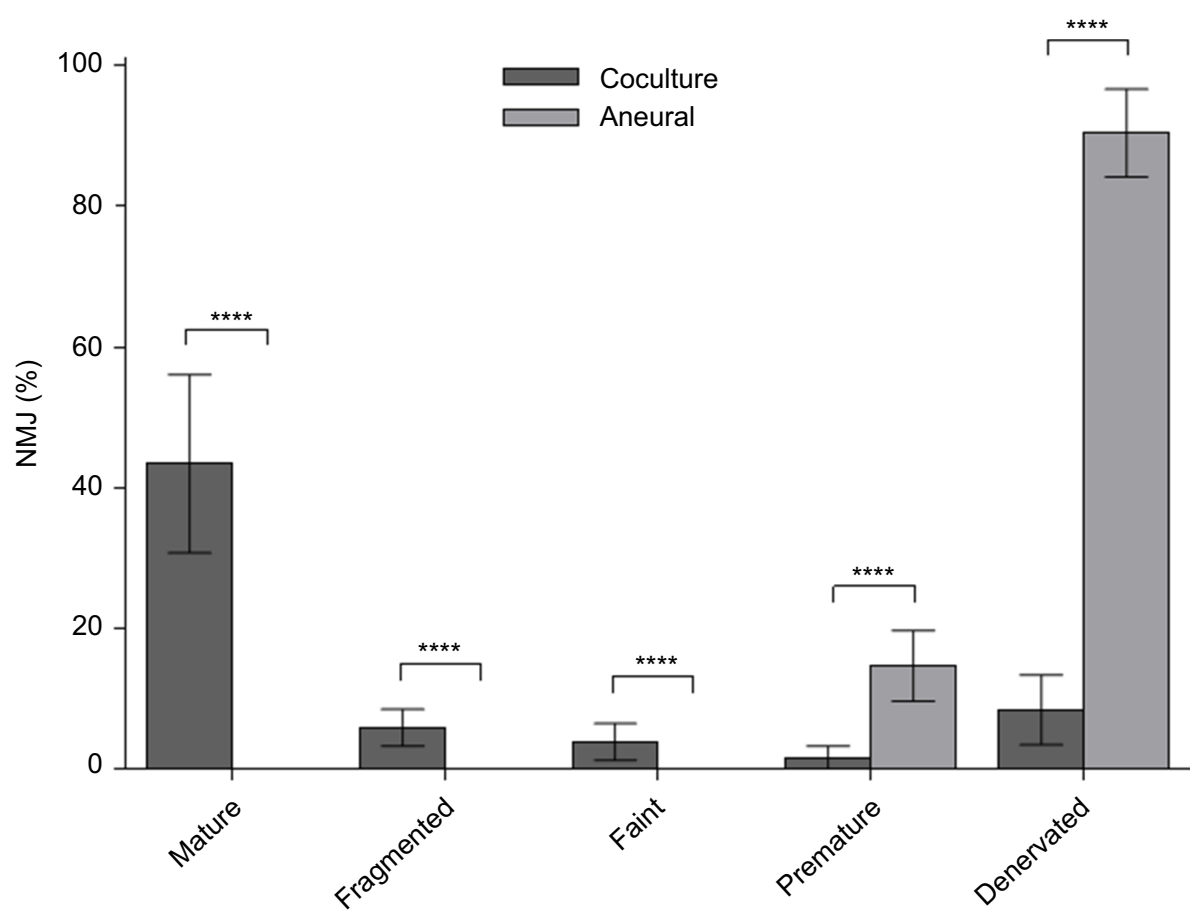

Figure 3 Percentage of various neuromuscular junction (NMJ) morphologies at day 14.

Notes: Cocultured and aneurally cultured myotubes. Results are expressed as mean $\pm S D, n=4$, analyzed with unpaired $t$-test; $* * * * P<0.000$ I.
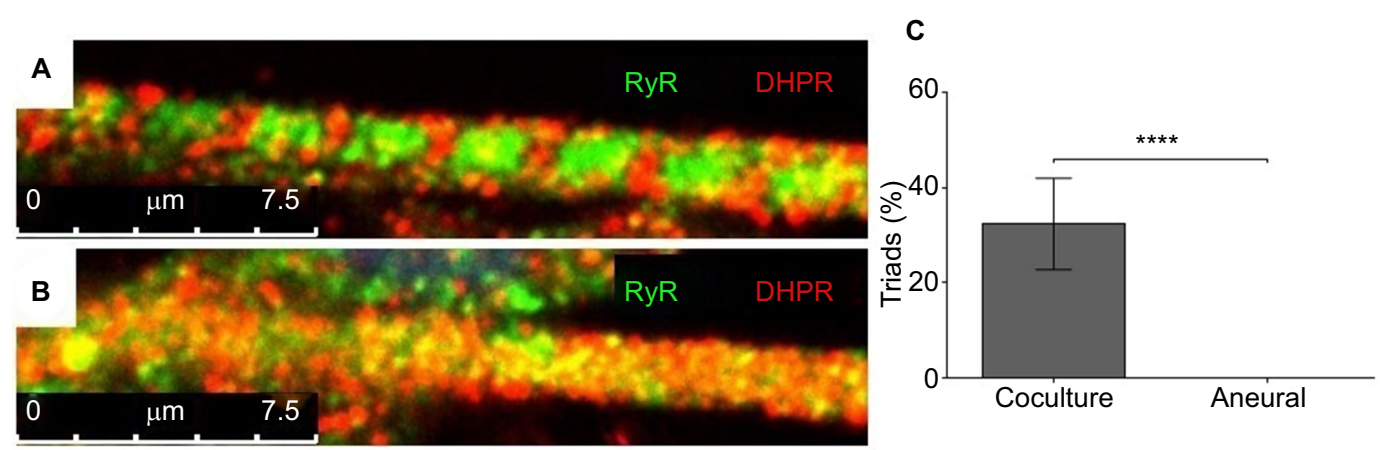

Figure 4 Characterization of myotubes at day 14.

Notes: Representative images of $(\mathbf{A})$ a myotube cocultured with embryonic rat spinal cord and (B) an aneurally cultured myotube. Myotubes were stained for ryanodine receptor (RyR) (green) and dihydropyridine receptor (DHPR) (red); scale bars $=7.5 \mu \mathrm{m}$. (C) The percentage of myotubes with triads from cocultured and aneurally cultured myotubes. Results are expressed as mean $\pm S D, n=10$, analyzed with unpaired $t$-test; $* * * * P<0.0001$.

that successfully produced abundant functional NMJs, as reflected by the structural differentiation and coordinated contractions of myotubes. This coculture model can be used as a model platform to test the efficacy of agents to modulate NMJ structure and function. This may be particularly useful in studies on NM diseases.

Neurons generated in this coculture model were derived from $\sim 1-2 \mathrm{~mm}$ transversally sliced spinal cord explants with intact DRGs. ${ }^{9,21}$ Preliminary experiments with mechanically disassociated embryo spinal cords to create a neuronal cell suspension, or coculture of myoblasts with the ventral horn only without the DRGs, showed poorer results than the coculture with entire rat embryonic spinal cord explants. The poorer result was reflected by a delayed initiation of spontaneous myotube contractions, higher incidence of arrhythmic contractions, lower contraction frequency, and lower abundance of NMJs that were poorly developed or had a failed junction assembly in comparison to the myoblast plus complete embryonic spinal cord segment coculture (data not shown). While this suggests that both motor and sensory neurons ${ }^{22}$ are required for correct innervation of myotubes and NMJ formation, it is more likely that the 
progenitor and supporting cell types, such as glial cells, in the DRGs are crucial for correct innervation. Indeed, Schwann cells perform vital functions in motoneuron development, differentiation, and maintaining NMJ integrity. ${ }^{23}$ Subsequently, we cocultured myoblasts with rat embryonic spinal cord explants.

In line with previous observations, ${ }^{24}$ we found that innervated myotubes in our coculture system were in an advanced state of differentiation and exhibited endogenously stimulated contractility, something not seen in the aneurally cultured SkMCs. The innervation appears to be important, as while treatment of aneurally cultured human myotubes with the secretome of a rat-nerve/human-muscle coculture secretome did increase AChR clustering, it failed to induce advanced myotube differentiation and contractile activity. ${ }^{25,26}$ In fact, early research found that myotubes that failed to become innervated within a nerve-muscle coculture system not only failed to differentiate, but eventually even deteriorated, despite nerve-derived secretions in the culture environment. ${ }^{27}$

In our coculture model, myotube contractions were observed as early as 72 hours post coculture, which we believe is the first time contractile functionality has been observed in any nerve-muscle coculture system. This indicates that functional NMJs were already present at this time point, as myotubes require nervous input to induce contraction. ${ }^{28}$ Over time, the contraction frequency increased and the contractions occurred synchronously by day 7 (Video S1). At day 14 , the cocultured innervated myotubes also displayed the regular appearance, every $2.4 \mu \mathrm{m}$, of transversal triads, peripherally located nuclei and actin-myosin striations, all morphological characteristics of advanced differentiation seen in vivo. ${ }^{29,30}$ The advanced differentiation of innervated myotubes illustrates the strength of this coculture system over typical aneural in vitro SkMC cultures, which fail to achieve advanced stages of development. Our coculture model thus provides a promising tool to elucidate the significance of the integrity of the NMJ in conditions such as NM disorders.

Our coculture model not only resembled the in vivo skeletal muscle more than aneurally cultured SkMC, but was also simple and reproducibly generated large quantities of functional NMJs. In contrast to our simple model, which already has functional NMJs after 3 days of coculture, other nerve-muscle coculture models require various culture media formulations to separately differentiate myotubes and motoneurons for at least 10 days before co-culturing can even start, ${ }^{31}$ and need another 21 days of co-culturing before NMJs form. ${ }^{5}$ These lengthy protocols lead to avoidable postponements, possible unintended variation to experimental procedures, and a larger risk of contamination. In our model, however, coculture can start immediately, and after only 3 days, viable NMJs are already detectable.

In addition, other nerve-muscle coculture systems require serum or trophic factors, such as brain-derived neurotrophic factor or insulin-like growth factor-1, to induce spontaneous myotube contractions, myotube innervation, and functional NMJ formation. ${ }^{5,31-33}$ Our coculture media were devoid of serum, neurotrophic factors, and growth factors, which will drastically reduce experimental variability. The simplicity of our system makes it ideal for high-throughput research of agents that may affect NMJ formation and myotube differentiation.

High throughput is also facilitated by the fact that a single pregnant rat can yield up to 100 embryo spinal cord explants. This also reduces between-experiment variation as the same spinal cord can be used for different experimental conditions and time points. Although our cocultures were characterized on day 14 , preliminary viability experiments revealed that persistent myotube contractions continued to occur as late as day 30, with the potential for further extended viability. Even though the lack of extended viability of other nerve--muscle models ${ }^{34}$ has been improved, ${ }^{32}$ they still only generated immature NMJs with a speckled AChR morphology and myotubes without advanced differentiation. Clearly, our system is an improvement on these coculture systems as we did see advanced myotube differentiation and a large number of functional NMJs.

Another advantage of our heterologous coculture model is that antibodies specific for rat or human can be used to study both presynaptic and postsynaptic regions of the NMJ. One such application is illustrated by the exploitation of speciesspecific immunocytochemical staining against acetylcholinesterase $(\mathrm{AChE})$ to differentiate between $\mathrm{AChE}$ originating from the human SkMCs and rat neuronal cells at the NMJ. ${ }^{35}$ Alternative chimeric cocultures have demonstrated similar advantages. For instance, a coculture model consisting of chick SkMCs innervated by rat neurons was stained using species-specific antibodies to determine that agrin, which is a vital element in the development of the NMJ, is secreted from both SkMCs and motoneurons into the synaptic cleft of NMJs. ${ }^{36}$ Fully human homologous hESC/hiPSC-derived SkMC and motoneuron coculture models would be of great interest. However, these systems are still in their infancy and require significant optimization before they parallel the formation of functional NMJs and express the level of differentiation demonstrated in our coculture model. 


\section{Conclusion}

We have developed an easy and reproducible serum-free coculture of immortalized human myoblasts and embryonic rat spinal cord explants that induces a rapid formation of functional NMJs. The model can be a used as a high-throughput platform to assess the impact of interventions on NM integrity and drug testing.

\section{Acknowledgments}

This work was sponsored by the School of Healthcare Science, Faculty of Science and Engineering, Manchester Metropolitan University (Manchester, UK). Thank you to the Lumley Trust, which paid for the animal costs.

\section{Author contributions}

JS, AF, AJR, HD, and NAS developed the study concept. All the authors contributed to experimental procedures. JS, AF, and NAS carried out the investigation. AJR, JSM, HD, and NAS supervised the study. JS, HD, APL, and NAS wrote the original draft of the manuscript. JS, MAAS, JSM, HD, and NAS reviewed and edited the draft manuscript. All authors contributed to data analysis and drafting or revising the article, gave final approval of the version to be published, and agree to be accountable for all aspects of the work.

\section{Disclosure}

The authors report no conflicts of interest in this work.

\section{References}

1. van der Worp HB, Howells DW, Sena ES, et al. Can animal models of disease reliably inform human studies? PLoS Med. 2010;7(3):e1000245.

2. Haase G. Motor neuron diseases: cellular and animal models. In: Meyers RA, editor. Reviews in Cell Biology and Molecular Medicine. Weinheim: Wiley-VCH Verlag GmbH \& Co. KGaA; 2006.

3. Prather RS, Lorson M, Ross JW, Whyte JJ, Walters E. Genetically engineered pig models for human diseases. Annu Rev Anim Biosci. 2013;1(1):203-219.

4. Suuronen EJ, Mclaughlin CR, Stys PK, Nakamura M, Munger R, Griffith M. Functional innervation in tissue engineered models for in vitro study and testing purposes. Toxicol Sci. 2004;82(2):525-533.

5. Demestre M, Orth M, Föhr KJ, et al. Formation and characterisation of neuromuscular junctions between hiPSC derived motoneurons and myotubes. Stem Cell Res. 2015;15(2):328-336.

6. Umbach JA, Adams KL, Gundersen CB, Novitch BG. Functional neuromuscular junctions formed by embryonic stem cell-derived motor neurons. PLoS One. 2012;7(5):e36049.

7. Harper JM, Krishnan C, Darman JS, et al. Axonal growth of embryonic stem cell-derived motoneurons in vitro and in motoneuron-injured adult rats. Proc Natl Acad Sci USA. 2004;101(18):7123-7128.

8. Guo X, Greene K, Akanda N, et al. In vitro differentiation of functional human skeletal myotubes in a defined system. Biomater Sci. 2014;2(1): 131-138.

9. Arnold A-S, Christe M, Handschin C. A functional motor unit in the culture dish: co-culture of spinal cord explants and muscle cells. $J$ Vis Exp. 2012;62(62):3616.
10. Rumsey JW, Das M, Stancescu M, Bott M, Fernandez-Valle C, Hickman JJ. Node of Ranvier formation on motoneurons in vitro. Biomaterials. 2009;30(21):3567-3572.

11. Mouly V, Aamiri A, Périé S, et al. Myoblast transfer therapy: is there any light at the end of the tunnel? Acta Myol. 2005;24(2):128-133.

12. Webster C, Blau HM. Accelerated age-related decline in replicative life-span of Duchenne muscular dystrophy myoblasts: implications for cell and gene therapy. Somat Cell Mol Genet. 1990;16(6):557-565.

13. Tanaka A, Woltjen K, Miyake K, et al. Efficient and reproducible myogenic differentiation from human iPS cells: prospects for modeling Miyoshi myopathy in vitro. PLoS One. 2013;8(4):e61540.

14. Stockmann M, Linta L, Föhr KJ, et al. Developmental and functional nature of human iPSC derived motoneurons. Stem Cell Rev. 2013;9(4):475-492.

15. Li XJ, Du ZW, Zarnowska ED, et al. Specification of motoneurons from human embryonic stem cells. Nat Biotechnol. 2005;23(2):215-221.

16. Sanes JR, Lichtman JW. Development of the vertebrate neuromuscular junction. Аnпи Rev Neurosci. 1999;22:389-442.

17. Low LK, Cheng HJ. Axon pruning: an essential step underlying the developmental plasticity of neuronal connections. Philos Trans $R$ Soc Lond B Biol Sci. 2006;361(1473):1531-1544.

18. Marty I, Robert M, Villaz M, et al. Biochemical evidence for a complex involving dihydropyridine receptor and ryanodine receptor in triad junctions of skeletal muscle. Proc Natl Acad Sci USA. 1994;91(6): 2270-2274.

19. Rios E, Brum G. Involvement of dihydropyridine receptors in excitationcontraction coupling in skeletal muscle. Nature. 1987;325(6106): 717-720.

20. Coronado R, Morrissette J, Sukhareva M, Vaughan DM. Structure and function of ryanodine receptors. Am J Physiol. 1994;266(6 Pt 1):C1485-C1504.

21. Kobayashi T, Askanas V, Engel WK. Human muscle cultured in monolayer and cocultured with fetal rat spinal cord: importance of dorsal root ganglia for achieving successful functional innervation. J Neurosci. 1987;7(10):3131-3141.

22. Mears SC, Frank E. Formation of specific monosynaptic connections between muscle spindle afferents and motoneurons in the mouse. $J$ Neurosci. 1997;17(9):3128-3135.

23. Riethmacher D, Sonnenberg-Riethmacher E, Brinkmann V, Yamaai T, Lewin GR, Birchmeier C. Severe neuropathies in mice with targeted mutations in the ErbB3 receptor. Nature. 1997;389(6652): 725-730.

24. Feher J, editor. The neuromuscular junction and excitation-contraction coupling. In: Quantitative Human Physiology. 2nd ed. Boston: Academic Press; 2017:318-333.

25. Bandi E, Jevšek M, Mars T, et al. Neural agrin controls maturation of the excitation-contraction coupling mechanism in human myotubes developing in vitro. Am J Physiol Cell Physiol. 2008;294(1): C66-C73.

26. Arnold A-S, Gueye M, Guettier-Sigrist S, et al. Reduced expression of nicotinic AChRs in myotubes from spinal muscular atrophy I patients. Lab Investig. 2004;84(10):1271-1278.

27. Askanas V, Kwan H, Alvarez RB, et al. De novo neuromuscular junction formation on human muscle fibres cultured in monolayer and innervated by foetal rat spinal cord: ultrastructural and ultrastructural-cytochemical studies. J Neurocytol. 1987;16(4):523-537.

28. Hong IHK, Etherington SJ, editors. Neuromuscular junction. In: eLS. Vol. 1. Chichester: John Wiley \& Sons; 2011.

29. Bruusgaard JC, Liestøl K, Ekmark M, Kollstad K, Gundersen K. Number and spatial distribution of nuclei in the muscle fibres of normal mice studied in vivo. J Physiol. 2003;551(2):467-478.

30. Shadrin IY, Khodabukus A, Bursac N. Striated muscle function, regeneration, and repair. Cell Mol Life Sci. 2016;73(22):4175-4202.

31. Guo X, Gonzalez M, Stancescu M, Vandenburgh HH, Hickman JJ. Neuromuscular junction formation between human stem cell-derived motoneurons and human skeletal muscle in a defined system. Biomaterials. 2011;32(36):9602-9611. 
32. das M, Rumsey JW, Bhargava N, Stancescu M, Hickman JJ. A defined long-term in vitro tissue engineered model of neuromuscular junctions. Biomaterials. 2010;31(18):4880-4888.

33. Rumsey JW, das M, Bhalkikar A, Stancescu M, Hickman JJ. Tissue engineering the mechanosensory circuit of the stretch reflex arc: sensory neuron innervation of intrafusal muscle fibers. Biomaterials. 2010;31(32):8218-8227.

34. das M, Rumsey JW, Gregory CA, et al. Embryonic motoneuron-skeletal muscle co-culture in a defined system. Neuroscience. 2007;146(2): 481-488.

35. Jevsek M, Mars T, Mis K, Grubic Z. Origin of acetylcholinesterase in the neuromuscular junction formed in the in vitro innervated human muscle. Eur J Neurosci. 2004;20(11):2865-2871.

36. Lieth E, Fallon JR. Muscle agrin: neural regulation and localization at nerve-induced acetylcholine receptor clusters. J Neurosci. 1993;13(6):2509-2514.
37. Mamchaoui K, Trollet C, Bigot A, et al. Immortalized pathological human myoblasts: towards a universal tool for the study of neuromuscular disorders. Skelet Muscle. 2011;1(1):34.

38. Valdez G, Tapia JC, Kang H, et al. Attenuation of age-related changes in mouse neuromuscular synapses by caloric restriction and exercise. Proc Natl Acad Sci USA. 2010;107(33):14863-14868.

39. Lee KY, Li M, Manchanda M, et al. Compound loss of muscleblindlike function in myotonic dystrophy. EMBO Mol Med. 2013;5(12): 1887-1900.

40. Kummer TT, Misgeld T, Lichtman JW, Sanes JR. Nerve-independent formation of a topologically complex postsynaptic apparatus. $J$ Cell Biol. 2004;164(7):1077-1087.

41. Sahashi K, Hua Y, Ling KK, et al. Tsunami: an antisense method to phenocopy splicing-associated diseases in animals. Genes Dev. 2012;26(16):1874-1884. 


\section{Supplementary material}

Video SI Phase-contrast video-micrograph of spontaneously contracting human myotubes that were cocultured with embryonic rat spinal cord explants at day 7. Representative video of innervated myotubes contracting within the coculture system without external stimulation. Scale bar $=100 \mu \mathrm{m}$.

Stem Cells and Cloning: Advances and Applications is an international, peer-reviewed, open access journal. Areas of interest in stem cell research include: Embryonic cell stems; Adult stem cells; Blastocysts; Cordblood stem cells; Stem cell transformation and culture; Therapeutic cloning; Umbilical cord blood and bone marrow cells; Laboratory, animal and human therapeutic studies; Philosophical and ethical issues related to stem cell research. This journal is indexed on CAS. The manuscript management system is completely online and includes a quick and fair peer-review system. Visit http://www.dovepress.com/ testimonials.php to read real quotes from published authors..

Submit your manuscript here: https://www.dovepress.com/stem-cells-and-cloning-advances-and-applications-journal 